\title{
Lorentz symmetry violation in QCD and the frustration of asymptotic freedom
}

\author{
I. T. Drummond $\odot^{*}$ \\ Department of Applied Mathematics and Theoretical Physics, Centre for Mathematical Sciences, \\ Wilberforce Road, Cambridge, England CB3 OWA, United Kingdom
}

(Received 20 January 2020; accepted 18 March 2020; published 8 April 2020)

\begin{abstract}
We study the effect of Lorentz symmetry violation (LSV) on the behavior at high energy of $S U(N)$ gauge theory with quarks in the fundamental representation. The approach is similar to that for QED treated in a previous paper. In contrast to QED, standard Lorentz invariant QCD is asymptotically free. Our aim is to explore the structure of the renormalization group at high energy and hence weak coupling without requiring the Lorentz symmetry breaking to be small. The simplest type of LSV leaves the theory invariant under a subgroup of the Lorentz group that preserves a (timelike) 4-vector. We examine this case in detail and find that asymptotic freedom is frustrated. That is, at sufficiently high energy, the running coupling constant attains a minimum value before increasing again, while the LSV parameter increases without bound.
\end{abstract}

DOI: 10.1103/PhysRevD.101.085004

\section{INTRODUCTION}

Lorentz symmetry violation (LSV) in QED has been studied by a number of authors concerned with its consistency with causality, unitarity [1-3], the structure of asymptotic states, and renormalization theory [4-6]. In previous papers $[7,8]$, we studied some of these issues in QED starting with a premetric formulation $[9,10]$ based on an action

$$
S=-\frac{1}{8} \int d^{4} x U^{\mu \nu \sigma \tau} F_{\mu \nu}(x) F_{\sigma \tau}(x)
$$

where $F_{\mu \nu}(x)$ is the standard electromagnetic field tensor and the (constant) background tensor $U^{\mu \nu \sigma \tau}$ has the same symmetry properties as the Riemann tensor in General Relativity, namely

$$
U^{\mu \nu \sigma \tau}=-U^{\nu \mu \sigma \tau}=U^{\sigma \tau \mu \nu}
$$

and

$$
U^{\mu \nu \sigma \tau}+U^{\mu \sigma \tau \nu}+U^{\mu \tau \nu \sigma}=0 .
$$

This latter condition excludes parity violation. An outcome of the analysis was that even when the Lorentz symmetry violation is not constrained to be small the behavior of the

\footnotetext{
*itd@damtp.cam.ac.uk
}

Published by the American Physical Society under the terms of the Creative Commons Attribution 4.0 International license. Further distribution of this work must maintain attribution to the author(s) and the published article's title, journal citation, and DOI. Funded by SCOAP ${ }^{3}$. renormalized theory in the infrared limit is dominated by the fixed point at zero coupling in a manner consistent with Lorentz symmetry. That is, at a sufficiently large scale in spacetime Lorentz symmetry reemerges. This is consistent with related earlier work [11-13].

In this paper, we study a QCD type model with $S U(N)$ gauge symmetry. In addition to the gauge field, we include a quark field that transforms under the fundamental representation of $S U(N)$. A closely related model is investigated in Ref. [14]. The significance of such a theory is that it exhibits asymptotic freedom; that is, its behavior at high energy is controlled, at least in the standard case of Lorentz invariance, by a weak coupling fixed point $[15,16]$. Our aim here is to investigate the manner in which asymptotic freedom is modified by the presence of Lorentz symmetry violation. An investigation with similar aims, in particular comparing QED and QCD, is presented in Ref. [17]. Although we look in detail only at the simplest type of LSV, we set out the general theory in a manner parallel to Ref. [7] in order to clarify the logical structure of the argument. This prepares a framework for analyses of more complex models.

In the obvious generalization of the case of QED, we take the action for the $S U(N)$ gauge field to be

$$
S_{g}=-\frac{1}{8} \int d^{4} x U^{\mu \nu \sigma \tau} F_{a \mu \nu}(x) F_{a \sigma \tau}(x),
$$

where $F_{a \mu \nu}(x)$ is the standard gauge field tensor transforming according to the orthogonal representation of $S U(N)$. For a general choice of $U^{\mu \nu \sigma \tau}$, this action, although gauge invariant, is not in general Lorentz invariant. Lorentz 
invariance with respect to a metric $g^{\mu \nu}$ can be recovered by choosing

$$
U^{\mu \nu \sigma \tau}=g^{\mu \sigma} g^{\nu \tau}-g^{\nu \sigma} g^{\mu \tau}
$$

Although there is a priori no metric in the general case with LSV, there is nevertheless, as argued in Ref. [7], a preferred metric $g^{\mu \nu}$ that allows us to decompose $U^{\mu \nu \sigma \tau}$ in the following way,

$$
U^{\mu \nu \sigma \tau}=g^{\mu \sigma} g^{\nu \tau}-g^{\nu \sigma} g^{\mu \tau}-C^{\mu \nu \sigma \tau},
$$

where the tensor $C^{\mu \nu \sigma \tau}$ has the same symmetries as the Weyl tensor in General Relativity. That is,

$$
C^{\mu \nu \sigma \tau}=-C^{\nu \mu \sigma \tau}=C^{\sigma \tau \mu \nu}
$$

and

$$
C^{\mu \nu \sigma \tau}+C^{\mu \sigma \tau \nu}+C^{\mu \tau \nu \sigma}=0
$$

In addition, it satisfies the trace condition

$$
g_{\mu \sigma} C^{\mu \nu \sigma \tau}=0 .
$$

We refer to $C^{\mu \nu \sigma \tau}$ as a Weyl-like tensor (WLT). It follows that the WLT determines the nature of the LSV. As in the case of QED, the possible types of LSV can be determined by applying the Petrov classification to the WLT [18]. A useful approach to the Petrov scheme is contained in Refs. [19,20]. Its application in QED with LSV is presented in Ref. [7]. There are six cases, conventionally labeled O, N, D, I, II, and III. Each case has a canonical form for the WLT [21].

Class $\mathrm{O}$ corresponds to the case $C^{\mu \nu \sigma \tau}=0$, which for pure gauge theory implies no LSV. However, as in the case of QED [7], the quark field can engender LSV in the model through its contribution to vacuum polarization provided the associated metric for quark propagation shares with the gluon metric an invariance under a subgroup of the Lorentz group that is the little group of the given 4-vector [22]. The 4-vector can be timelike, spacelike, or lightlike (with respect to both metrics). The timelike case implies that there is a reference frame in which the theory is invariant under rotations of the spatial axes. This is the case we study in detail. However, it is convenient to set out the scheme for quantizing and renormalizing the theory in a general form. Canonical forms for the WLT in other Petrov classes and the implications for the vector meson dispersion relations are the same as those for photons in QED as described in detail in Ref. [7].

It should be emphasized that our approach is entirely consistent with and indeed is part of the Standard Model Extension introduced and developed in Ref. [4]. In the simplest terms, there is a notational correspondence $g^{\mu \nu} \rightarrow \eta^{\mu \nu}, \bar{g}^{\mu \nu} \rightarrow \eta^{\mu \nu}+c^{\mu \nu}$, and $C^{\mu \nu \rho \tau} \rightarrow \kappa_{G}^{\mu \nu \rho \tau}$ from this paper to Ref. [4]. However, there is a small explicit difference beyond notation. The aim in Ref. [4] was to set out the departure from Lorentz invariance in a manner appropriate for phenomenological applications, for which it is natural to assume it is very small. Our aim is to compute as far as possible without assuming that the LSV is small, so it is not convenient to split $\bar{g}^{\mu \nu}$ as in the above correspondence. There is a further difference between our approach and that of Ref. [4] in that we allow both $g^{\mu \nu}$ and $\bar{g}^{\mu \nu}$ to undergo a renormalization in dealing with UV divergences. The reason is, as explained in Sec. V, to permit a simple multiplicative renormalization of the gauge fields. However, the equivalence of the two approaches can be made evident by a linear change of coordinates that restores $g^{\mu \nu}$ to a standard form which we can choose to be $\eta^{\mu \nu}$. This change of coordinates of course involves UV divergences through the perturbative expansion in the coupling constant. The structure of this argument is completely evident in the application of the theory to the case of Petrov class O.

\section{GAUGE FIXING AND GHOST FIELDS}

In terms of the vector gauge fields $A_{a \mu}(x)$, the tensor fields are given by

$$
F_{a \mu \nu}(x)=\partial_{\mu} A_{a \nu}(x)-\partial_{\nu} A_{a \mu}(x)+g f_{a b c} A_{b \mu}(x) A_{c \nu}(x),
$$

where $g$ is the gauge field coupling constant and $f_{a b c}$ are the structure constants of $S U(N)$. In order to deal with the gauge invariance of the action for the vector fields in Eq. (4), we follow the approach in Ref. [7] and impose the gauge condition

$$
\Lambda^{\mu \nu} \partial_{\mu} A_{a \nu}(x)=0
$$

Here, $\Lambda^{\mu \nu}$ is a metriclike tensor, which we will find it convenient to distinguish from $g^{\mu \nu}$ because the two tensors behave differently under the renormalization procedure. We are therefore led to add a gauge fixing term to the action of the form

$$
S_{g f}=\frac{1}{2} \int d^{4} x\left(\Lambda^{\mu \nu} \partial_{\mu} A_{a \nu}(x)\right)^{2}
$$

In addition and in contrast to the case of QED [7], we must introduce anticommuting ghost fields $c_{a}(x)$ and $\bar{c}_{a}(x)$ in order to construct in the standard way the FadeevPopov determinant in the path integral formalism for the computation of Green's functions in the gauge theory. We therefore complete the action for the gauge theory by adding a term, 


$$
S_{g h}=-\int d^{4} x \bar{c}_{a}(x) \Lambda^{\mu \nu} \partial_{\mu} D_{a b \nu} c_{b}(x)
$$

Here, $D_{a b \nu}=\delta_{a b} \partial_{\nu}+g f_{a b c} A_{c \nu}(x)$ is the gauge covariant derivative for the ghost fields. The complete action for the theory is $S$, where

$$
S=S_{g}+S_{g f}+S_{g h}
$$

\section{FEYNMAN RULES}

The Feynman rules for the theory can be read off from the action $S$ in the standard way. They are in certain respects analogous to the corresponding rules for Bimetric QED [7].

\section{A. Feynman propagator}

The Feynman propagator, illustrated in Fig. 1(a), is

$$
\Delta_{F a b \mu \nu}(q)=-i \delta_{a b} M_{\mu \nu}(q)
$$

where $M_{\mu \nu}(q)$ is the matrix inverse to $M^{\mu \nu}(q)$ and

$$
M^{\mu \nu}(q)=\left(U^{\mu \alpha \nu \beta}+\Lambda^{\mu \alpha} \Lambda^{\nu \beta}\right) q_{\alpha} q_{\beta} .
$$

More explicitly,

$$
M^{\mu \nu}(q)=q^{2} g^{\mu \nu}-q^{\mu} q^{\nu}+Q^{\mu} Q^{\nu}-C^{\mu \alpha \nu \beta} q_{\alpha} q_{\beta} .
$$

Here, $Q^{\mu}=\Lambda^{\mu \alpha} q_{\alpha}$. It is easy to verify that when $C^{\mu \alpha \nu \beta}$ vanishes and $\Lambda^{\mu \nu}=g^{\mu \nu}$ this reduces to the standard Lorentz invariant form. Following the analysis for the photon propagator in Ref. [7], we first introduce $\mathcal{M}^{\mu \nu}(q)$, which is the form taken by $M^{\mu \nu}(q)$ when indeed $\Lambda^{\mu \nu}$ is replaced by $g^{\mu \nu}$, that is,

$$
\mathcal{M}^{\mu \nu}(q)=q^{2} g^{\mu \nu}-C^{\mu \alpha \nu \beta} q_{\alpha} q_{\beta} .
$$

The inverse matrix is $\mathcal{M}_{\mu \nu}(q)$ (see Ref. [7]), and it can be used to construct $M_{\mu \nu}(q)$ in the form

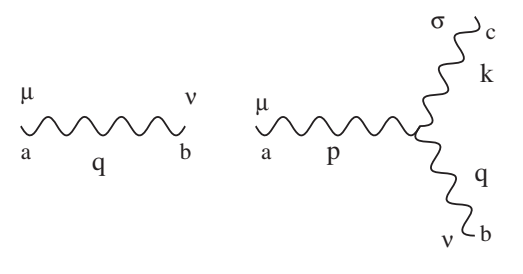

(a)

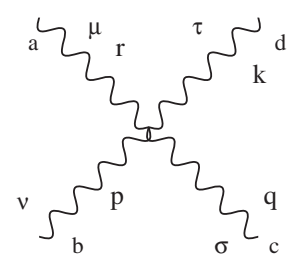

(c)
FIG. 1. Gluons (a) propagator, (b) 3-vertex, and (c) 4-vertex. 4-momenta inward.
$M_{\mu \nu}(q)=\left(\delta_{\mu}^{\sigma}-\frac{q_{\mu} Q^{\sigma}}{q \cdot Q}\right) \mathcal{M}_{\sigma \tau}(q)\left(\delta_{\nu}^{\tau}-\frac{Q^{\tau} q_{\nu}}{q \cdot Q}\right)+\frac{q_{\mu} q_{\nu}}{(q \cdot Q)^{2}}$

A careful analysis [7] shows that the Feynman propagator has the same vector meson poles as $\mathcal{M}_{\mu \nu}(q)$ together with the ghost poles at $q \cdot Q=\Lambda^{\mu \nu} q_{\mu} q_{\nu}=0$. It is straightforward to check that were we to set $\Lambda^{\mu \nu}=g^{\mu \nu}$ then we would find

$$
M_{\mu \nu}(q)=\mathcal{M}_{\mu \nu}(q)
$$

\section{B. Gluon vertices}

The three-gluon vertex, Fig. 1(b), is

$$
V_{a b c}^{\mu \nu \sigma}=-g f_{a b c}\left(p_{\rho} U^{\rho \mu \nu \sigma}+q_{\rho} U^{\rho \nu \sigma \mu}+k_{\rho} U^{\rho \sigma \mu \nu}\right) .
$$

For the four-gluon vertex, Fig. 1(c), we have

$$
\begin{aligned}
V^{\mu \nu \sigma \tau}= & -i g^{2}\left(U^{\mu \nu \sigma \tau} f_{h a b} f_{h c d}+U^{\mu \sigma \tau \nu} f_{h a c} f_{h b d}\right. \\
& \left.+U^{\mu \tau \nu \sigma} f_{h a d} f_{h b c}\right) .
\end{aligned}
$$

Of course, momentum conservation is enforced at each vertex. Again, it is easy to verify that in the absence of LSV these vertices reduce to standard form (see, for example, Ref. [23]).

\section{Ghost propagator and vertex}

The Feynman propagator for the ghost fields, Fig. 2(a), is

$$
\Delta_{a b}^{(g h)}(p)=i \frac{\delta_{a b}}{P \cdot p},
$$

where $P^{\mu}=\Lambda^{\mu \nu} p_{\nu}$ and $P . p=P^{\mu} p_{\mu}=\Lambda^{\mu \nu} p_{\mu} p_{\nu}$. Momentum follows the ghost direction. From this, it is obvious that the mass-shell condition for the ghosts is $P . p=\Lambda^{\mu \nu} p_{\mu} p_{\nu}=0$.

The vertex coupling the ghosts to the vector field is indicated in Fig. 2(b) and has the form

$$
V_{a b c}^{(g h) \mu}=-g f_{a b c} \Lambda^{\mu \nu} p_{\nu} .
$$

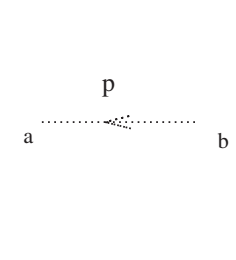

(a)

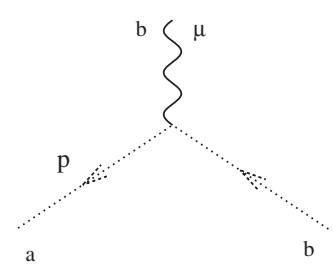

(b)
FIG. 2. The ghost propagator is indicated in (a), and the vertex for coupling to gluons is in (b). 


\section{QUARK FIELD}

The model can be extended by including one or more spinor fields each transforming under $S U(N)$. For simplicity, we will consider the case of one such field. Modifications can be added later. The action for this field is $S_{q u}$, where

$$
S_{q u}=\int d^{4} x \bar{\psi}(x)\left(i \bar{\gamma}^{\mu} D_{\mu}[A]-m\right) \psi(x) .
$$

Here,

$$
\bar{\gamma}^{\mu}=\gamma^{a} \bar{e}_{a}^{\mu},
$$

where $\bar{e}_{a}^{\mu}$ is the vierbein associated with the spinor field and $\left\{\gamma^{a}\right\}$ are the standard Dirac $\gamma$-matrices. The metric associated with the spinor field is $\bar{g}^{\mu \nu}=\eta^{a b} \bar{e}^{\mu}{ }_{a} \bar{e}^{\nu}{ }_{b}$. This is a second source of LSV in the model. Of course, $D_{\mu}[A]$ is the gauge covariant derivative appropriate to the spinor field. The Feynman propagator for the spinor field is indicated in Fig. 3(a),

$$
S_{F}(p)=\frac{i}{\bar{\gamma}^{\mu} p_{\mu}-m}
$$

and the coupling to the gauge field is indicated in Fig. 3(b),

$$
\mathcal{V}_{a}^{\mu}=i g \bar{\gamma}^{\mu} t_{a}
$$

Here, $t_{a}$ is the $S U(N)$ generator appropriate to the representation of the spinor field (see Ref. [23]).

We use dimensional regularization [24] in order to deal with the ultraviolet divergences of the theory. The Feynman diagrams for the perturbation series for the Green's functions of the theory are evaluated in $n$ dimensions. All the parameters of the theory, coupling constant, mass, metrics, and WLT are subject to corrections involving UV divergences. We denote the bare quantities with an extra suffix 0 . Each bare parameter is expanded in terms of a renormalized coupling constant $g$. For example,

$$
g \rightarrow g_{0}=\mu^{(4-n) / 2} g\left(1+\sum_{k=1}^{\infty} g^{(k)} g^{2 k}\right) .
$$

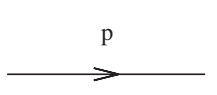

(a)

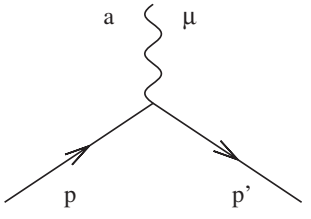

(b)
FIG. 3. The quark propagator is indicated in (a), and the quark gluon coupling is in (b).
Here, $\mu$ is the scale associated with the renormalized coupling $g$ [24]. The coefficients $g^{(k)}$ depend on the dimension $n$ and exhibit poles of various orders at $n=4$. For example, $g^{(1)}$ has a simple pole at $n=4$. The other parameters are similarly replaced by bare versions that are expanded in powers of the renormalized coupling $g$,

$$
\begin{aligned}
m_{0} & =m\left(1+\sum_{k=1}^{\infty} b^{(k)} g^{2 k}\right) \\
g_{0}^{\mu \nu} & =g^{\mu \nu}+\sum_{k=1}^{\infty} g^{(k) \mu \nu} g^{2 k} \\
\bar{e}_{0 a}^{\mu} & =\bar{e}^{\mu}{ }_{a}+\sum_{k=1}^{\infty} \bar{e}^{(k) \mu}{ }_{a} g^{2 k} \\
C_{0}^{\mu \nu \sigma \tau} & =C^{\mu \nu \sigma \tau}+\sum_{k=1}^{\infty} C^{(k) \mu \nu \sigma \tau} g^{2 k} \\
\Lambda_{0}^{\mu \nu} & =\Lambda^{\mu \nu}+\sum_{k=1}^{\infty} \Lambda^{(k) \mu \nu} g^{2 k} .
\end{aligned}
$$

Again, the coefficients in the various expansions exhibit poles at $n=4$. Note that we are free to assume that $\operatorname{det} g_{0}^{\mu \nu}=\operatorname{det} g^{\mu \nu}=-1$. This implies that

$$
g_{\mu \nu} g^{(1) \mu \nu}=0 .
$$

\section{PERTURBATIVE CALULATIONS AT ONE LOOP}

\section{A. Renormalization of gauge field propagator}

The vacuum polarization tensor $\Sigma_{a b}^{\mu \nu}(q)$ determines the renormalization properties of the (inverse) gluon propagator $\Delta_{a b}^{\mu \nu}(q)$. The (one-loop) diagrams that contribute to $i \Sigma_{a b}^{\mu \nu}(q)$ are shown in Fig. 4 (see Ref. [23]). We have, to $O\left(g^{2}\right)$,

$$
\Delta_{0 a b}^{\mu \nu}(q)=\Delta_{F 0 a b}^{\mu \nu}(q)+i \Sigma_{a b}^{\mu \nu}(q) .
$$

Here, the inverse Feynman propagator is expressed in terms of the bare parameters,

$\Delta_{F 0 a b}^{\mu \nu}(q)=-i \delta_{a b}\left\{\left(g_{0}^{\mu \nu} g_{0}^{\alpha \beta}-g_{0}^{\mu \beta} g_{0}^{\nu \alpha}+\Lambda_{0}^{\mu \beta} \Lambda_{0}^{\nu \alpha}-C_{0}^{\mu \alpha \nu \beta}\right) q_{\alpha} q_{\beta}\right\}$.

In principle, the contributions to the vacuum polarization are also computed from the appropriate Feynman diagrams using the bare parameters. However, our calculation will be of $O\left(g^{2}\right)$, so we need only use the lowest order expansions in Eqs. (30). This amounts to using the renormalized parameters in the vertices and propagators when computing $i \Sigma_{a b}^{\mu \nu}(q)$. In addition, the UV divergences of $i \Sigma_{a b}^{\mu \nu}(q)$ occur only in the lowest terms in its Taylor expansion in $q_{\alpha}$. On general grounds, then, we can exhibit the UV divergences to $O\left(g^{2}\right)$ by writing 


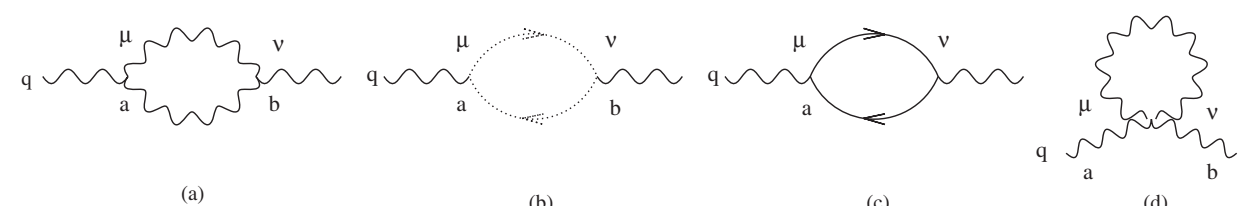

FIG. 4. Contributions to vacuum polarization from (a) gluons, (b) ghosts, (c) quarks, and (d) the gluon loop.

$$
i \Sigma_{a b}^{\mu \nu}(q)=i \delta_{a b} \frac{g^{2}}{n-4} W^{\mu \alpha \nu \beta} q_{\alpha} q_{\beta}+O\left(q^{4}\right),
$$

where the $O\left(q^{4}\right)$ terms are UV finite. The tensor $W^{\mu \alpha \nu \beta}$ has the same symmetry properties as the Riemann tensor. Hence, in a standard fashion, it can be expressed in the form

$$
\begin{aligned}
W^{\mu \alpha \nu \beta}= & \left\{\frac{1}{12} W\left(g^{\mu \nu} g^{\alpha \beta}-g^{\mu \beta} g^{\nu \alpha}\right)\right. \\
& +\frac{1}{2}\left(V^{\mu \nu} g^{\alpha \beta}+g^{\mu \nu} V^{\alpha \beta}-V^{\mu \beta} g^{\nu \alpha}-g^{\mu \beta} g^{\nu \alpha}\right) \\
& \left.-V^{\mu \alpha \nu \beta}\right\},
\end{aligned}
$$

where

$$
\begin{aligned}
W & =W^{\alpha \beta} g_{\alpha \beta}, \\
W^{\alpha \beta} & =W^{\mu \alpha \nu \beta} g_{\mu \nu}, \\
V^{\alpha \beta} & =W^{\alpha \beta}-\frac{1}{4} W g^{\alpha \beta} .
\end{aligned}
$$

We have also

$$
\begin{aligned}
V^{\alpha \beta} g_{\alpha \beta} & =0, \\
V^{\mu \alpha \nu \beta} g_{\alpha \beta} & =0 .
\end{aligned}
$$

It follows that $V^{\mu \alpha \nu \beta}$, having the appropriate symmetries and trace properties, is a WLT. In Eqs. (36) and (37), we have used the four-dimensional decomposition, which is adequate when computing the residues of the pole at $n=4$. The type of Lorentz symmetry breaking exhibited by the model can be specified by means of the Petrov classification of Weyl tensors. We will return to this point later.

From Eqs. (29), (30), and (33), we see that

$$
\Delta_{F 0 a b}^{\mu \nu}(q)=\Delta_{F a b}^{\mu \nu}(q)+\delta \Delta_{F a b}^{\mu \nu}(q),
$$

where $\Delta_{F a b}^{\mu \nu}(q)$ is obtained from $\Delta_{F 0 a b}^{\mu \nu}(q)$ by replacing the bare parameters with their renormalized versions and

$$
\begin{aligned}
\delta \Delta_{F a b}^{\mu \nu}(q)= & -\delta_{a b} i g^{2}\left\{g^{\alpha \beta} g^{(1) \mu \nu}+g^{(1) \alpha \beta} g^{\mu \nu}-g^{\mu \alpha} g^{(1) \nu \beta}\right. \\
& -g^{(1) \mu \alpha} g^{\nu \beta}+\Lambda^{\alpha \beta} \Lambda^{(1) \mu \nu}+\Lambda^{(1) \alpha \beta} \Lambda^{\mu \nu} \\
& \left.-\Lambda^{\mu \alpha} \Lambda^{(1) \nu \beta}-\Lambda^{(1) \mu \alpha} \Lambda^{\nu \beta}-C^{(1) \mu \alpha \nu \beta}\right\} q_{\alpha} q_{\beta} .
\end{aligned}
$$

The renormalization parameters are fixed by requiring that the renormalization of $\Delta_{0 a b}^{\mu \nu}(q)$ reduces to an overall multiplicative factor, that is,

$\Delta_{0 a b}^{\mu \nu}(q)=\left(1-\frac{1}{12} \frac{g^{2}}{n-4} W\right)\left(\Delta_{F a b}^{\mu \nu}(q)+O\left(q^{4}\right)\right)$.

This is achieved by requiring that

$$
\begin{aligned}
g^{(1) \mu \nu} & =\frac{1}{2} \frac{1}{n-4} V^{\mu \nu}, \\
\Lambda^{(1) \mu \nu} & =\frac{1}{24} \frac{1}{n-4} W \Lambda^{\mu \nu}, \\
C^{(1) \mu \alpha \nu \beta} & =\frac{1}{n-4}\left(V^{\mu \alpha \nu \beta}-C^{\mu \alpha \nu \beta}\right) .
\end{aligned}
$$

Note that the results in Eqs. (41) do imply that $g^{(1) \mu \nu} g_{\mu \nu}=0$. Furthermore, the renormalization of $\Lambda_{0}^{\mu \nu}$ is multiplicative and involves the field renormalization factor $Z^{1 / 2}$, where

$$
Z^{1 / 2}=1+\frac{1}{24} \frac{g^{2}}{n-4} W .
$$

This is not necessarily true of the metric renormalization. For this reason, it is conceptually convenient to distinguish the bare metric from the bare ghost metric. However, at the level of one loop in perturbation theory, it is possible and convenient to allow the two metrics to coincide. Higher order calculations may require the maintenance of the distinction, imposed order by order, between the two metrics.

\section{B. Renormalization of quark propagator}

The renormalization of the quark propagator $i S_{0}(p)$ proceeds along similar lines. We have

$$
S_{0}^{-1}(p)=S_{F 0}^{-1}(p)+\Sigma(p),
$$

where

$$
\begin{gathered}
S_{F 0}^{-1}(p)=\bar{\gamma}_{0}^{\mu} p_{\mu}+m_{0}, \\
\bar{\gamma}_{0}^{\mu}=\gamma^{a} e_{0 a}^{\mu}
\end{gathered}
$$

and $\gamma^{a}$ are standard Dirac $\gamma$-matrices. The quark propagator is implicitly a unit operator in $S U(N)$ space. The selfenergy amplitude $i \Sigma(p)$ can be calculated (at one loop) from the diagram in Fig. 5 using the Feynman rules with 


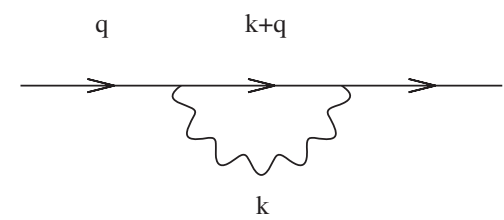

FIG. 5. Quark self-energy.

renormalized parameters. The UV divergences can be exposed in the Taylor expansion

$$
\Sigma(p)=\Sigma(0)+\Sigma^{\mu}(0) p_{\mu}+O\left(p^{2}\right)
$$

where the contribution $O\left(p^{2}\right)$ is finite at $n=4$, and we have

$$
\Sigma(0)=m \frac{g^{2} \sigma}{n-4}
$$

and

$$
\Sigma^{\mu}(0)=\frac{g^{2}}{n-4} H_{\nu}^{\mu} \bar{\gamma}^{\nu}
$$

We write

$$
H^{\mu}{ }_{\nu}=\frac{1}{n} H \delta_{\nu}^{\mu}+h^{\mu}{ }_{\nu},
$$

where $h^{\mu}{ }_{\nu}=0$. The term $H$ determines the quark propagator renormalization $Z_{q}$, while $h^{\mu}{ }_{\nu}$ fixes the counterterm in $\bar{e}_{0 a}^{\mu}$. More explicitly, we have, taking account only of the poles at $n=4$,

$$
\begin{gathered}
Z_{q}=1-\frac{1}{4} \frac{g^{2}}{n-4} H, \\
g^{2} b^{(1)}=\frac{g^{2}}{n-4} \sigma+\frac{1}{4} \frac{g^{2}}{n-4} H,
\end{gathered}
$$

and

$$
g^{2} \bar{e}^{(1) \mu}{ }_{b} \bar{e}^{b}+\frac{g^{2}}{n-4} h^{\mu}{ }_{\nu}=0
$$

That is,

$$
g^{2} \bar{e}^{(1) \mu}{ }_{a}+\frac{g^{2}}{n-4} h_{\nu}^{\mu} \bar{e}^{\nu}{ }_{a}=0
$$

implying that, to $O\left(g^{2}\right)$,

$$
\bar{g}_{0}^{\mu \nu}=\bar{g}^{\mu \nu}-\frac{g^{2}}{n-4}\left(h^{\mu}{ }_{\rho} \bar{g}^{\rho \nu}+h_{\rho}^{\nu} \bar{g}^{\rho \mu}\right) .
$$

\section{Renormalization of coupling constant}

The coupling constant renormalization is most easily followed by considering the quark-gluon vertex. The relevant diagrams are shown in Fig. 6 and yield contributions to the truncated three-point function that render it

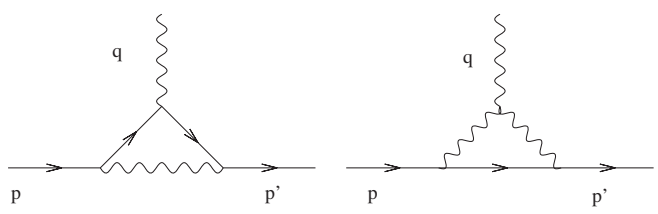

FIG. 6. Quark gluon coupling diagram.

finite after appropriate field renormalizations. We can anticipate the nature of the divergences by examining the bare vertex $\mathcal{V}_{0 a}^{\mu}=i g_{0} \bar{\gamma}_{0}^{\mu} t_{a}$. From Eqs. (29) and (30), we have

$\left.\mathcal{V}_{0 a}^{\mu}=i g_{0} \bar{\gamma}_{0}^{\mu} t_{a}=i \mu^{(4-n) / 2} g\left(1+g^{2} g^{(1)}\right)\left(\bar{e}_{b}^{\mu}+g^{2} \bar{e}^{(1) \mu}{ }_{b}\right)\right) \gamma^{b} t_{a}$.

That is,

$\mathcal{V}_{0 a}^{\mu}=i \mu^{(4-n) / 2} g\left(\left(1+g^{2} g^{(1)}\right) \delta_{\nu}^{\mu}-\frac{g^{2}}{n-4} h^{\mu}{ }_{\nu}\right) \bar{\gamma}^{\nu} t_{a}$.

The point we make here is that the renormalization of the quark vierbein enters into the vertex calculation and the contribution $\mathcal{V}_{a}^{\mu}\left(p, p^{\prime}\right)$ to the three point function from the diagrams in Fig. 6 must be consistent with this. We can expect then that $\mathcal{V}_{a}^{\mu}\left(p, p^{\prime}\right)$ will (at zero external momenta) have the form

$\mathcal{V}_{a}^{\mu}(0,0)=i \mu^{(4-n) / 2} g\left(\frac{g^{2}}{n-4} K \delta_{\nu}^{\mu} t_{a}+\frac{g^{2}}{n-4} h^{\mu}{ }_{\nu}\right) \bar{\gamma}^{\nu} t_{a}$.

This will be verified in particular calculations. The truncated three point function $\mathcal{V}_{a}^{(3) \mu}\left(p, p^{\prime}\right)$ will, at zero external momentum, satisfy

$\mathcal{V}_{a}^{(3) \mu}(0,0)=i \mu^{(4-n) / 2} g\left(1+g^{2} g^{(1)}+\frac{g^{2}}{n-4} K\right) \bar{\gamma}^{\mu} t_{a}$.

Finally, $g^{(1)}$ is determined by requiring that the right side of this equation is rendered finite by extracting the field renormalization factors $Z_{q}^{-1} Z^{-1 / 2}$, implying

$$
g^{2} g^{(1)}=\frac{g^{2}}{n-4}\left(\frac{1}{4} H-\frac{1}{24} W-K\right) .
$$

We will look at this in more detail when evaluating the vertex in the special case of LSV we consider below.

\section{RENORMALIZATION GROUP}

From the results in Sec. V, we can obtain the renormalization group ( $R G$ ) equations for the renormalized parameters to lowest nontrivial order in the coupling 
constant $g$. These are derived from the requirement that the bare parameters are independent of the renormalization scale $\mu$. For the coupling constant, we have

$$
\mu \frac{\partial}{\partial \mu} g_{0}=0 .
$$

From Eq. (57), we then obtain the renormalization group $\beta$-function

$\beta(\mu)=\mu \frac{\partial}{\partial \mu} g=-(2-n / 2) g-g^{3}\left(\frac{1}{4} H-\frac{1}{24} W-K\right)$.

Note that in deriving Eq. (61) from Eq. (60), we have ignored derivatives of $H, W$, and $K$ since they are of $O\left(g^{2}\right)$ and may, and indeed must, be ignored at one loop. Of course, the first term on the right of Eq. (61) vanishes in four dimensions.

Following the same principles, we obtain for the gluon metric

$$
\mu \frac{\partial}{\partial \mu} g^{\alpha \beta}=-\frac{1}{2} g^{2} V^{\alpha \beta}
$$

and

$$
\mu \frac{\partial}{\partial \mu} \bar{g}^{\alpha \beta}=g^{2}\left(h_{\rho}^{\alpha} \bar{g}^{\rho \beta}+h^{\beta}{ }_{\rho} \bar{g}^{\rho \alpha}\right) .
$$

We obtain also for the renormalized mass

$$
\mu \frac{\partial m}{\partial \mu}=-m g^{2}\left(\sigma+\frac{1}{4} H\right) .
$$

The renormalization scheme set out here can be applied to LSV associated with a WLT of any of the Petrov classes. However, even at one loop, the calculations are rather complex. Partly then for reasons of simplicity, we restrict attention in this paper to models in Petrov class O. The results nevertheless remain interesting.

\section{RENORMALIZATION FOR PETROV CLASS O}

As explained above, in the case of Petrov class $\mathrm{O}$, the LSV is due entirely to the difference between the light cone associated with the gluons and that associated with the quark field. That is, we are assuming that the tensor $C^{\mu \nu \sigma \tau}$ vanishes. This is possible if there is a reference frame in which rotational invariance holds simultaneously for both metrics. Given the symmetry properties of $C^{\mu \nu \sigma \tau}$, it is consistent with this rotational invariance only if it is null. Similar remarks apply to situations where the preserved subgroup of the Lorentz group, in a suitable frame, leaves invariant either a purely spacelike or lightlike vector. We will concentrate on the rotationally invariant case. We have for the gluon metric

$$
g^{\mu \nu}=\left(\begin{array}{cccc}
\alpha & 0 & 0 & 0 \\
0 & -\beta & 0 & 0 \\
0 & 0 & -\beta & 0 \\
0 & 0 & 0 & -\beta
\end{array}\right)
$$

and for the quark metric

$$
\bar{g}^{\mu \nu}=\left(\begin{array}{cccc}
\bar{\alpha} & 0 & 0 & 0 \\
0 & -\bar{\beta} & 0 & 0 \\
0 & 0 & -\bar{\beta} & 0 \\
0 & 0 & 0 & -\bar{\beta}
\end{array}\right) .
$$

We assume that

$$
\alpha \beta^{3}=\bar{\alpha} \bar{\beta}^{3}=1
$$

so that $\operatorname{det} g^{\mu \nu}=\operatorname{det} \bar{g}^{\mu \nu}=-1$. We have similar forms for the bare metrics $g_{0}^{\mu \nu}$ and $\bar{g}_{0}^{\mu \nu}$ in terms of the bare parameters $\left(\alpha_{0}, \beta_{0}\right)$ and $\left(\bar{\alpha}_{0}, \bar{\beta}_{0}\right)$ which have appropriate expansions in powers of the renormalized coupling. That is,

$$
\alpha_{0}=\alpha+\frac{g^{2}}{n-4} \alpha^{(1)}+\ldots
$$

together with similar expansions for the other bare parameters. It is convenient to relate the two metrics by setting $\bar{\alpha}=a \alpha$ and $\bar{\beta}=b \beta$ with the consequence that $a b^{3}=1$. Similar remarks hold in an obvious way for bare parameters $a_{0}$ and $b_{0}$. The significance then of $b$ is that in a coordinate frame in which the gluon metric is diagonal with entries $(1,-1,-1,-1)$ the quarks have a light cone associated with a velocity $c_{q}=b^{2}$. At appropriately high energies, we would expect that when $b>1$ the quarks travel faster than the gluons and slower when $b<1$.

\section{A. Vacuum polarization for Petrov class $O$}

In order to carry out the renormalization process, we assume the renormalized gauge metric satisfies (to oneloop order) $\Lambda^{\mu \nu}=g^{\mu \nu}$. The vanishing of $C^{\mu \nu \sigma \tau}$ then yields for the renormalized gluon propagator

$$
M_{\mu \nu}(q)=\frac{g_{\mu \nu}}{q^{2}} .
$$

The vertex factors, as mentioned above, acquire the standard form for the gauge theory without (at this stage) any LSV. We can therefore use the discussion of nonAbelian gauge theories in Ref. [23] to evaluate the contributions from the diagrams in Figs. 4(a), 4(b), and 4(d) to obtain the gauge field contribution to the UV divergence at one loop as 


$$
\begin{aligned}
i \Sigma_{a b}^{(g) \mu \nu}(q)= & -\frac{10}{3} C_{2}(G) \frac{i g^{2}}{(4 \pi)^{2}} \delta_{a b}\left(g^{\mu \nu} g^{\alpha \beta}-g^{\mu \beta} g^{\alpha \nu}\right) q_{\alpha} q_{\beta} \\
& \times \frac{1}{n-4},
\end{aligned}
$$

where $C_{2}(G)$ is the value of the quadratic Casimir operator in the adjoint representation of $S U(N)$. As explained in Ref. [23], the diagram in Fig. 4(d) does not contribute to this pole residue. In a similar way, we can use the results in Ref. [23] to evaluate the quark contribution to the vacuum polarization from Fig. 4(c) provided we use $\bar{g}^{\mu \nu}$ as the appropriate metric. The result is

$$
i \Sigma_{a b}^{(q) \mu \nu}(q)=\frac{4}{3} \frac{i g^{2}}{(4 \pi)^{2}} \delta_{a b}\left(\bar{g}^{\mu \nu} \bar{g}^{\alpha \beta}-\bar{g}^{\mu \beta} \bar{g}^{\alpha \nu}\right) \frac{1}{n-4} .
$$

From Eq. (36), we see that contribution to the tensor $W^{\mu \alpha \nu \beta}$ from the gluon field term is

$$
W^{(g) \mu \alpha \nu \beta}=-\frac{1}{(4 \pi)^{2}} \frac{10}{3} C_{2}(G)\left(g^{\mu \nu} g^{\alpha \beta}-g^{\mu \alpha} g^{\beta \nu}\right)
$$

and

$$
W^{(g)}=-\frac{40}{(4 \pi)^{2}} C_{2}(G)
$$

It is immediately obvious $V^{(g) \mu \nu}$ vanishes as does $V^{(g) \mu \alpha \nu \beta}$. From the quark field term, we have

$$
W^{(q) \mu \alpha \nu \beta}=\frac{1}{(4 \pi)^{2}} \frac{4}{3}\left(\bar{g}^{\mu \nu} \bar{g}^{\alpha \beta}-\bar{g}^{\mu \beta} \bar{g}^{\alpha \nu}\right) .
$$

We have then

$$
W^{(q)}=\frac{8}{(4 \pi)^{2}} \frac{\bar{\beta}}{\beta}\left(\frac{\bar{\alpha}}{\alpha}+\frac{\bar{\beta}}{\beta}\right)=\frac{8}{(4 \pi)^{2}} b(a+b),
$$

and

$$
V^{(q) \alpha \beta}=\frac{2}{(4 \pi)^{2}} b(a-b)\left(\begin{array}{cccc}
\alpha & 0 & 0 & 0 \\
0 & \beta / 3 & 0 & 0 \\
0 & 0 & \beta / 3 & 0 \\
0 & 0 & 0 & \beta / 3
\end{array}\right) .
$$

It follows that

$$
W=W^{(g)}+W^{(q)}=\frac{8}{(4 \pi)^{2}}\left(b(a+b)-5 C_{2}(G)\right) .
$$

Of course,

$$
V^{\mu \nu}=V^{(q) \mu \nu} .
$$

Finally, then, from Eqs. (41) and (78), we have

$$
\alpha^{(1)}=\frac{1}{(4 \pi)^{2}} \frac{1}{n-4} b(a-b) \alpha=\frac{1}{(4 \pi)^{2}} \frac{1}{n-4}\left(\frac{1}{b^{2}}-b^{2}\right) \alpha,
$$

that is,

$$
\alpha_{0}=\alpha\left(1+\frac{g^{2}}{(4 \pi)^{2}} \frac{1}{n-4}\left(\frac{1}{b^{2}}-b^{2}\right)\right),
$$

and

$$
\beta_{0}=\beta\left(1-\frac{1}{3} \frac{g^{2}}{(4 \pi)^{2}} \frac{1}{n-4}\left(\frac{1}{b^{2}}-b^{2}\right)\right)
$$

In the limit of Lorentz invariance, $a=b=1$, and we have $\alpha_{0}=\alpha$ and $\beta_{0}=\beta$. The field renormalization factor is

$$
Z^{1 / 2}=1+\frac{1}{3} \frac{g^{2}}{(4 \pi)^{2}} \frac{1}{n-4}\left(\left(\frac{1}{b^{2}}+b^{2}\right)-5 C_{2}(G)\right) .
$$

\section{B. Quark self-energy for Petrov class O}

The results for the calculations in the previous section, because each term involves only a single metric, can be read off from standard results (see Ref. [23]). However, the one-loop self-energy correction to the quark propagator is obtained from the Feynman rules applied to the diagram in Fig. 5. Both metrics are involved. A possible conflict with causality might be anticipated. This possibility has been discussed previously, and the conclusion is that in the present case there is no difficulty, as is confirmed directly in the calculations $[1-3,7,25]$. We have then

$$
i \Sigma(p)=-g^{2} C_{2}(N) \int \frac{d^{n} k}{(2 \pi)^{n}} \bar{\gamma}^{\mu} \frac{1}{\bar{\gamma}^{\alpha}(p+k)_{\alpha}-m} \bar{\gamma}^{\nu} \frac{g_{\mu \nu}}{k^{2}} .
$$

We require

$$
i \Sigma(0)=-g^{2} C_{2}(N) \int \frac{d^{n} k}{(2 \pi)^{n}} \bar{\gamma}^{\mu} \frac{1}{\bar{\gamma}^{\alpha} k_{\alpha}-m} \bar{\gamma}^{\nu} \frac{g_{\mu \nu}}{k^{2}},
$$

and

$$
\begin{aligned}
\left.i \frac{\partial}{\partial p_{\lambda}} \Sigma(p)\right|_{p=0} & =i \Sigma^{\lambda}(0) \\
& =g^{2} C_{2}(N) \int \frac{d^{n} k}{(2 \pi)^{n}} \bar{\gamma}^{\mu} \frac{1}{\bar{\gamma}^{\alpha} k_{\alpha}-m} \bar{\gamma}^{\lambda} \frac{1}{\bar{\gamma}^{\beta} k_{\beta}-m} \bar{\gamma}^{\nu} \frac{g_{\mu \nu}}{k^{2}} .
\end{aligned}
$$


The evaluation of these terms is along the same lines as the corresponding calculation for QED in Ref. [7]. We find for the UV poles at $n=4$

$$
i \Sigma(0)=i m \frac{4 g^{2}}{(4 \pi)^{2}} C_{2}(N) \frac{1}{n-4} \frac{a+3 b}{\sqrt{b}(\sqrt{a}+\sqrt{b})},
$$

with the result

$$
\sigma=\frac{4}{(4 \pi)^{2}} C_{2}(N) \frac{1+3 b^{4}}{b^{2}\left(1+b^{2}\right)}
$$

We have also

$$
i \Sigma^{0}(0)=i \frac{4 g^{2}}{(4 \pi)^{2}} C_{2}(N) \frac{1}{n-4} \frac{a-3 b}{(\sqrt{a}+\sqrt{b})^{2}} \bar{\gamma}^{0},
$$

$i \Sigma^{j}(0)=-i \frac{4}{3} \frac{g^{2}}{(4 \pi)^{2}} C_{2}(N) \frac{1}{n-4} \frac{(a+b)(2 \sqrt{a}+\sqrt{b})}{\sqrt{b}(\sqrt{a}+\sqrt{b})^{2}} \bar{\gamma}^{j}$.

Using $a=b^{-3}$, we find for the mass renormalization

$$
b^{(1)}=-\frac{4}{(4 \pi)^{2}} C_{2}(N) \frac{1}{n-4} \frac{1+3 b^{4}}{b^{2}\left(1+b^{2}\right)} .
$$

We find also

$$
H=-\frac{4}{(4 \pi)^{2}} C_{2}(N) \frac{2\left(1-b^{2}+2 b^{4}\right)}{b^{2}\left(1+b^{2}\right)}
$$

and

$h_{\rho}^{\lambda}=\frac{4}{(4 \pi)^{2}} C_{2}(N) \frac{\left(1-b^{2}\right)\left(1+3 b^{2}+4 b^{4}\right)}{2 b^{2}\left(1+b^{2}\right)^{2}} \mathcal{T}_{\rho}^{\lambda}$.

Here, $\mathcal{T}_{\rho}^{\lambda}$ is a traceless diagonal matrix with entries $(1,-1 / 3,-1 / 3,-1 / 3)$. It then follows that

$\bar{\beta}_{0}=\bar{\beta}\left(1+\frac{1}{3} \frac{4 g^{2}}{(4 \pi)^{2}} C_{2}(N) \frac{1}{n-4} \frac{\left(1-b^{2}\right)\left(1+3 b^{2}+4 b^{4}\right)}{b^{2}\left(1+b^{2}\right)^{2}}\right)$.

Combining this with Eq. (81), we obtain

$$
\begin{aligned}
b_{0}= & b\left(1+\frac{1}{3} \frac{g^{2}}{(4 \pi)^{2}} \frac{1}{n-4} \frac{\left(1-b^{2}\right)}{b^{2}}\right. \\
& \left.\times\left[\left(1+b^{2}\right)+4 C_{2}(N) \frac{\left(1+3 b^{2}+4 b^{4}\right)}{\left(1+b^{2}\right)^{2}}\right]\right) .
\end{aligned}
$$

\section{Coupling constant renormalization for Petrov class $\mathbf{O}$}

The one-loop diagrams in Fig. 6 yield the coupling constant renormalization. For the computation of the UV pole divergence, it is sufficient to calculate the vertex with $p=p^{\prime}=0$. From the first diagram, we obtain

$$
\mathcal{V}_{a}^{\lambda}(0,0)=g^{3} t_{b} t_{a} t_{b} g_{\mu \nu} I^{\mu \lambda \nu},
$$

where

$$
I^{\mu \lambda \nu}=\int \frac{d^{n} k}{(2 \pi)^{n}} \bar{\gamma}^{\mu} \frac{1}{\bar{\gamma}^{\alpha} k_{\alpha}-m} \bar{\gamma}^{\lambda} \frac{1}{\bar{\gamma}^{\beta} k_{\beta}-m} \bar{\gamma}^{\nu} \frac{1}{k^{2}} .
$$

On omitting terms that do not contribute to the UV divergence, we have

$$
I^{\mu \lambda \nu}=\bar{\gamma}^{\mu} \bar{\gamma}^{\alpha} \bar{\gamma}^{\lambda} \bar{\gamma}^{\beta} \bar{\gamma}^{\nu} T_{\alpha \beta}
$$

then,

$$
T_{\alpha \beta}=\int \frac{d^{n} k}{(2 \pi)^{n}} \frac{k_{\alpha} k_{\beta}}{\left(\bar{g}^{\alpha^{\prime} \beta^{\prime}} k_{\alpha^{\prime}} k_{\beta^{\prime}}-m^{2}\right)^{2} k^{2}} .
$$

The result using $t_{b} t_{a} t_{b}=\left(C_{2}(N)-C_{2}(G) / 2\right) t_{a}$ and $a b^{3}=1$ is

$\mathcal{V}_{a}^{0}(0,0)=4 \frac{i g^{3}}{(4 \pi)^{2}} \frac{1}{n-4}\left(C_{2}(N)-C_{2}(G) / 2\right) \frac{1-3 b^{4}}{\left(1+b^{2}\right)^{2}} t_{a} \bar{\gamma}^{0}$.

We have also

$$
\begin{aligned}
\mathcal{V}_{a}^{j}(0,0)= & -\frac{4}{3} \frac{i g^{3}}{(4 \pi)^{2}} \frac{1}{n-4}\left(C_{2}(N)_{-} C_{2}(G) / 2\right) \\
& \times \frac{\left(1+b^{4}\right)\left(2+b^{2}\right)}{b^{2}\left(1+b^{2}\right)^{2}} t_{a} \bar{\gamma}^{j} .
\end{aligned}
$$

In the case of the second diagram, we note that in the limit of zero external momenta the internal three-gluon vertex reduces to

$$
\begin{aligned}
V_{a b c}^{\lambda \mu \nu} & =-g f_{a b c} k_{\rho}\left(U^{\rho \nu \lambda \mu}-U^{\rho \mu \nu \lambda}\right) \\
& =-g f_{a b c} k_{\rho}\left(2 g^{\rho \lambda} g^{\nu \mu}-g^{\rho \mu} g^{\nu \lambda}-g^{\rho \nu} g^{\mu \lambda}\right) .
\end{aligned}
$$

The contribution to the vertex becomes

$$
\begin{aligned}
\mathcal{V}_{a}^{\mu}(0,0)= & -\frac{1}{2} g^{3} t_{a} \bar{\gamma}^{\nu^{\prime}} \bar{\gamma}^{\alpha} \bar{\gamma}^{\mu^{\prime}} g_{\mu \mu^{\prime}} g_{\nu \nu^{\prime}} \\
& \times\left(2 g^{\rho \lambda} g^{\mu \nu}-g^{\rho \mu}-g^{\rho \nu} g^{\mu \lambda}\right) \tilde{T}_{\alpha \rho},
\end{aligned}
$$

where 


$$
\tilde{T}_{\alpha \rho}=\int \frac{d^{n} k}{(2 \pi)^{4}} \frac{k_{\alpha} k_{\rho}}{\left(\bar{g}^{\alpha^{\prime} \beta^{\prime}} k_{\alpha^{\prime}} k_{\beta^{\prime}}-m^{2}\right)\left(k^{2}\right)^{2}} .
$$

We have again omitted terms that do not contribute to the UV pole at $n=4$. The tensor $\tilde{T}_{\alpha \rho}$ is closely related to $T_{\alpha \rho}$ in Eq. (98). Finally, we have the contributions

$$
\mathcal{V}_{a}^{0}(0,0)=-4 \frac{i g^{3}}{(4 \pi)^{2}} C_{2}(G) \frac{1}{n-4} \frac{b^{2}\left(1+2 b^{2}\right)}{\left(1+b^{2}\right)^{2}} t_{a} \bar{\gamma}^{0}
$$

and

$\mathcal{V}_{a}^{j}(0,0)=-\frac{4}{3} \frac{i g^{3}}{(4 \pi)^{2}} C_{2}(G) \frac{1}{n-4} \frac{1+2 b^{2}+4 b^{4}+2 b^{6}}{b^{2}\left(1+b^{2}\right)^{2}} t_{a} \bar{\gamma}^{j}$.

Combining the two sets of results, we obtain

$\mathcal{V}_{a}^{0}(0,0)=4 \frac{i g^{3}}{(4 \pi)^{2}} \frac{1}{n-4} t_{a} \bar{\gamma}^{0}\left(C_{2}(N) \frac{1-3 b^{4}}{\left(1+b^{2}\right)^{2}}-\frac{1}{2} C_{2}(G)\right)$

and

$$
\begin{aligned}
\mathcal{V}_{a}^{j}(0,0)= & -\frac{4}{3} \frac{i g^{3}}{(4 \pi)^{2}} \frac{1}{n-4} t_{a} \bar{\gamma}^{j}\left(C_{2}(N) \frac{\left(1+b^{4}\right)\left(2+b^{2}\right)}{b^{2}\left(1+b^{2}\right)^{2}}\right. \\
& \left.+\frac{3}{2} C_{2}(G)\right) .
\end{aligned}
$$

This leads to

$$
\mathcal{V}_{a}^{\lambda}(0,0)=4 \frac{i g^{3}}{(4 \pi)^{2}} \frac{1}{n-4} t_{a} R_{\rho}^{\lambda} \bar{\gamma}^{\rho},
$$

where

$$
\begin{aligned}
R_{\rho}^{\lambda}= & -\frac{1}{2}\left(C_{2}(N) \frac{1-b^{2}+2 b^{4}}{b^{2}\left(1+b^{2}\right)}+C_{2}(G)\right) \delta_{\rho}^{\lambda} \\
& +\frac{1}{2} C_{2}(N) \frac{\left(1-b^{2}\right)\left(1+3 b^{2}+4 b^{4}\right)}{b^{2}\left(1+b^{2}\right)^{2}} \mathcal{T}^{\lambda} .
\end{aligned}
$$

As expected from Eq. (56), the second contribution to $R^{\lambda}{ }_{\rho}$ yields the correct term $\propto h_{\rho}^{\lambda}$. The bare coupling and the one-loop correction yield

$$
\begin{aligned}
& \mathcal{V}_{0 a}^{\lambda}(0,0)+\mathcal{V}_{a}^{\lambda}(0,0) \\
& =i \mu^{(4-n) / 2} g\left(1+g^{(1)} g^{2}-\frac{4 g^{2}}{(4 \pi)^{2}} \frac{1}{n-4} \frac{1}{2}\right. \\
& \left.\quad \times\left(C_{2}(N) \frac{1-b^{2}+2 b^{4}}{b^{2}\left(1+b^{2}\right)}+C_{2}(G)\right)\right) t_{a} \bar{\gamma}^{\lambda} .
\end{aligned}
$$

Thus, from Eq. (59), we find that

$$
K=-\frac{2}{(4 \pi)^{2}}\left(C_{2}(N) \frac{1-b^{2}+2 b^{4}}{b^{2}\left(1+b^{2}\right)}+C_{2}(G)\right) .
$$

The remaining UV divergences, as shown in Eq. (59), are removed by appropriate field renormalization factors and then finally by the pole in the coupling constant expansion. We have then from Eq. (110) the result for the $\beta$-function for the renormalized coupling

$$
\beta(g)=-(2-n / 2)-\frac{g^{3}}{(4 \pi)^{2}}\left(\frac{11}{3} C_{2}(G)-\frac{1}{3}\left(\frac{1}{b^{2}}+b^{2}\right)\right) .
$$

Obviously, the first term vanishes in four dimensions. The second term reduces to the standard answer when $b=1$ and there is no Lorentz symmetry breaking.

\section{Renormalization group for Petrov class $\mathbf{O}$}

In four dimensions then, the important renormalization group equations are for $g$ and $b$. They take the form

$$
\mu \frac{\partial g}{\partial \mu}=-\frac{g^{3}}{(4 \pi)^{2}}\left(\frac{11}{3} C_{2}(G)-\frac{1}{3}\left(\frac{1}{b^{2}}+b^{2}\right)\right),
$$

and

$\mu \frac{\partial b}{\partial \mu}=-\frac{1}{3} \frac{g^{2}}{(4 \pi)^{2}} \frac{1-b^{2}}{b^{2}}\left(1+b^{2}+4 C_{2}(N) \frac{1+3 b^{2}+4 b^{4}}{\left(1+b^{2}\right)^{2}}\right)$.

In general, the two variables influence one another as they evolve along the RG trajectory. However, some points in $(g, b)$-space are particularly significant. The Lorentz invariant situation $b=1$ is stable and is maintained under the RG. The coupling constant $g$ then runs to zero, its fixed point, in the standard way as $\mu$ rises to infinity. The rate at which $g$ drops is the result of a competition between the contributions of the gauge field and the quark field to the vacuum polarization. When we explore values of $b \neq 1$, we see that the effect of the quark field is enhanced with the result that $\beta(g)$ vanishes when $b$ satisfies

$$
11 C_{2}(G)-\frac{1}{b^{2}}-b^{2}=0 .
$$

That is,

$$
b=b_{ \pm}=\left(\frac{1}{2}\left(R \pm \sqrt{R^{2}-4}\right)\right)^{1 / 2},
$$

where $R=11 C_{2}(G)$. On these two lines $\partial b / \partial \mu$ remains nonvanishing. The RG trajectories cross the lines, and at the 
crossing point, the coupling constant attains a minimum value. It increases again as the scaling energy $\mu$ continues to increase.

If we modify the model so that it contains $n_{f}$ quarks, all sharing the same metric, then Eq. (113) becomes

$$
\mu \frac{\partial g}{\partial \mu}=-\frac{g^{3}}{(4 \pi)^{2}}\left(\frac{11}{3} C_{2}(G)-\frac{n_{f}}{3}\left(\frac{1}{b^{2}}+b^{2}\right)\right) .
$$

Equation (114) remains unchanged. The minimum of $g$ occurs when

$$
11 C_{2}(G)-n_{f}\left(\frac{1}{b^{2}}+b^{2}\right)=0
$$

that is, when $b=b_{ \pm}$, where now $R=11 C_{2}(G) / n_{f}$.

The RG equation for the quark mass is obtained from Eqs. (64) and (87). It is

$$
\mu \frac{\partial m}{\partial \mu}=-2 m \frac{g^{2}}{(4 \pi)^{2}} C_{2}(N) \frac{1+b^{2}+4 b^{4}}{b^{2}\left(1+b^{2}\right)} .
$$

\section{DISCUSSION}

The behavior of the RG trajectory described above shows that a frustration of asymptotic freedom can arise in the presence of LSV, at least in this model. The question arises as to whether it might be observable experimentally. There are several issues to be considered, for example the relationship of the lab frame to the gluon frame used in the above discussion. We set this matter provisionally aside, though it must ultimately be resolved, and assume that the lab frame is traveling slowly relative to the gluon frame. More significant is the size of the energy range implicit in the model. We introduce an initial energy $M_{I}$ and associated LSV parameter $b_{I}, Q C D$ coupling $\alpha_{I}=\alpha_{S}\left(M_{I}\right)$ and the energy $M_{\min }$ at which the coupling reaches its minimum value.

In a gesture toward "reality," we consider the case $S U(3)$ gauge theory, where $C_{2}(G)=3$ and $C_{2}(3)=4 / 3$ [23] with $n_{f}=6$ quarks. In this case, $b_{+}=2.3947$ and $b_{-}=0.4339$. The light cone of the quarks in the gluon frame at minimum coupling is $c_{q}=b_{+}^{2}=5.311$ for LSV with $b>1$ and $c_{q}=b_{-}^{2}=0.1882$ for LSV with $b<1$. These values for $c_{q}$ represent rather severe LSV.

In order that our asymptotic calculation is relevant, we must assume $M_{I}$ is sufficiently large and in particular is greater than the top quark mass, that is, $173 \mathrm{GeV}$. In this energy regime, there is no easy way to relate our calculation to low energy determinations of $\alpha_{S}(\mu)$. The story of the running coupling and its relationship to low energy phenomena and $\Lambda_{\mathrm{QCD}}$ is complicated. It is comprehensively reviewed in Ref. [26]. An important point is the subtraction scheme used to obtain finite results for physical quantities. We are using the minimal subtraction $(M S)$ scheme [24].
More widely used is the $\overline{\mathrm{MS}}$ scheme introduced in Ref. [27] to improve convergence. In that scheme, the strong coupling has an evaluation $\alpha_{S}\left(M_{Z} \simeq 90 \mathrm{GeV}\right) \simeq 0.12$. On the grounds that our calculation is exploratory, we feel justified in neglecting the difference in subtraction schemes and propose $\alpha_{S}\left(M_{I}\right)=0.1$ when $M_{I}=10^{2}-10^{3} \mathrm{GeV}$. The qualitative nature of the results is not altered by (relatively) small changes in our initial conditions.

With these initial conditions, the results for examples of the renormalization trajectory, obtained by numerical integration (second order Runge-Kutta (RK)) of Eqs. (117) and (114) are shown in Fig. 7. Obviously, the closer the initial value $b_{I}$ is to unity, the closer the renormalization group trajectory stays near the Lorentz symmetry line and the later it breaks away, heading for its minimum value. These results are illustrated in Fig. 8, which shows the connection between $\log _{10}\left(M_{\min } / M_{I}\right)$ and $b_{I}$. The smooth curve is obtained by fitting the rightmost point on the plot. Even for this implausibly high value $b_{I}=1.1$ at our initial energy scale $M_{I}=10^{2} \mathrm{GeV}$, we still find $M_{\min } \simeq 10^{25} M_{I}$. Tuning $b_{I}$ down to potentially more realistic values results in yet greater disparities in the orders of magnitude of $M_{\min }$ and experimentally attainable values for $M_{I}$. The conclusion must therefore be that for QCD with the known set of quarks there is little hope of observing any of the frustration of asymptotic freedom in accelerator experiments. However, the complex asymptotic behavior that we encounter in this model may have relevance to very high energy processes at very early times in the initiating big bang of the Universe. In view of the fact that the energy range associated with frustration of asymptotic freedom appears to lie well above the Planck mass $\left(M_{P} \simeq 10^{19} \mathrm{GeV}\right)$ where gravitational effects must become important, one might question its physical relevance. However, it may also be possible and would certainly be interesting to relate the behavior of $\alpha_{S}(M)$ when $M \simeq M_{P}$ to models of quantum gravity constructed with appropriate running LSV parameters $[28,29]$.

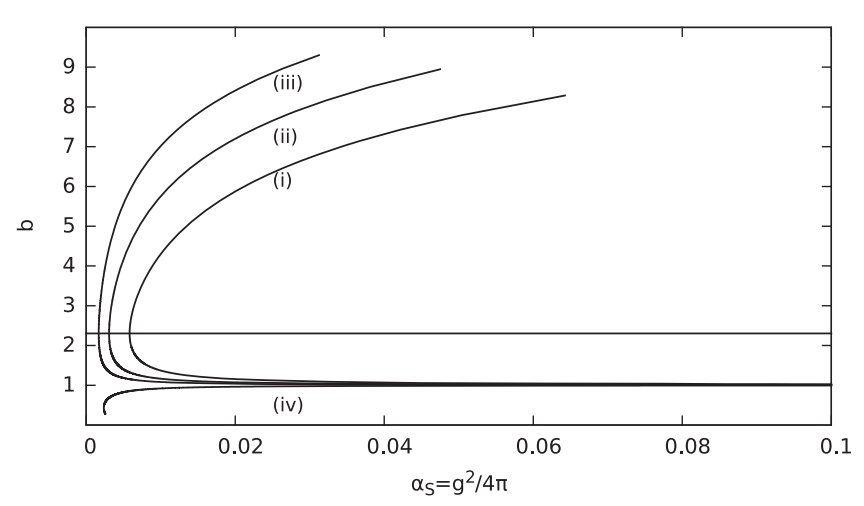

FIG. 7. The RG trajectories for $S U(3)$ with $n_{f}=6$ starting at $\alpha_{S}=0.1$. The initial values of $b$ are (a) 1.025, (b) 1.0125, (c) 1.00625, and (d) 0.99375 . The horizontal line is at $b=b_{+}=2.3047$. 


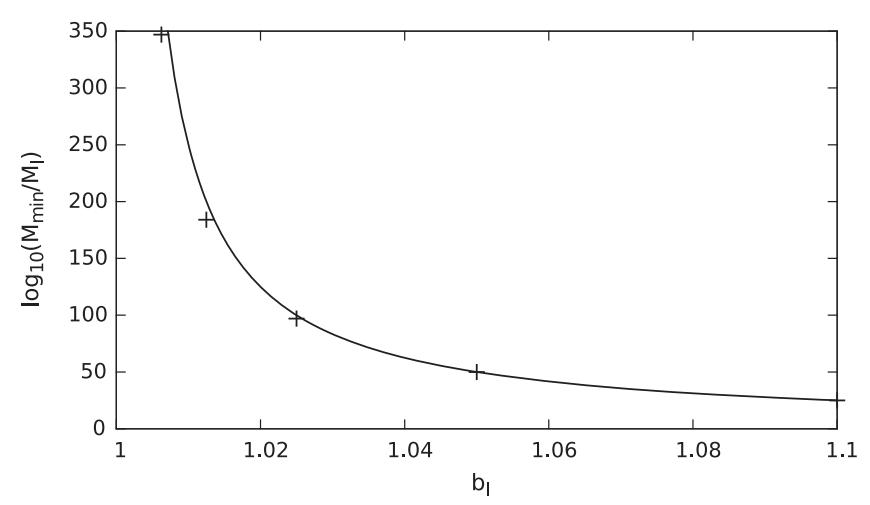

FIG. 8. Here, $M_{I}$ is the initial energy scale, $b_{I}$ is the corresponding LSV parameter, and $M_{\min }$ is the energy scale at which the running coupling attains a minimum. The results of the R-K integration are represented by crosses and compare well with the continuous curve $\log _{10}\left(M_{\min } / M_{I}\right)=2.5 /\left(b_{I}-1\right)$.

These considerations do not preclude the possibility of discovering LSV effects in an energy range for which $b$ remains close to unity. For example, if we set $b=1+x$ and assume $x$ is small, then to lowest order in $x$, Eqs. (117) and (114) become

$$
\begin{aligned}
& \mu \frac{\partial g}{\partial \mu}=-A \frac{g^{3}}{(4 \pi)^{2}}, \\
& \mu \frac{\partial x}{\partial \mu}=B \frac{g^{2}}{(4 \pi)^{2}} x,
\end{aligned}
$$

where $A=\left(11 C_{2}(G)-2 n_{f}\right) / 3$ and $B=4\left(1+4 C_{2}(N)\right) / 3$. In this approximation,

$$
\alpha_{S}(E)=\alpha_{I}\left(1+7 \frac{\alpha_{I}}{4 \pi} \log \frac{E}{M_{I}}\right)^{-1},
$$

the RG trajectories have the form

$$
b=1+x_{I}\left(\alpha_{S} / \alpha_{I}\right)^{-\kappa},
$$

where $x_{I}$ is the initial value of $x$ and $\kappa=-B / 2 A$. Equation (123) exhibits the instability at the fixed point $\left(\alpha_{S}, b\right)=(0,1)$. Depending on the sign selected for $x_{I}, b$ will either rise or fall from unity as $\alpha_{S}$ approaches zero. With our choice of parameters, we have $A=7$ and $B=$ 8.4444 with the result $\kappa=0.6031$. The dependence of $x$ on $\alpha_{S}$ is therefore relatively weak. When $\alpha_{S}$ decreases by an order of magnitude, $x$ only increases by a factor of roughly 4. A similar approximation for the renormalized mass $m$ yields

$$
\frac{m}{m_{I}}=\left(\frac{\alpha_{S}}{\alpha_{I}}\right)^{\tau}
$$

where $\tau=0.762$. In the asymptotic energy range then, the renormalized mass reduces, also relatively slowly, with a power of the renormalized coupling. If effective methods were developed for computing the structure and scattering of high energy particles in the model (see Refs. [30,31] for related discussions in QED and the Standard Model Extension), then possibly it could provide guidance for accelerator experiments and cosmic ray detectors investigating LSV phenomena in a high energy regime of PeV and beyond. For example, if we take (intuitively) the quark metric, $\operatorname{diag}(a,-b-b-b)$, as determining the dispersion relation for quark based states, it would become for a particle with mass $m$, energy $E$, and momentum $P$,

$$
a E^{2}-b P^{2}=m^{2} c_{q}^{4} \text {. }
$$

Combining the above results, we find for the velocity of quark based particles

$$
v=\frac{d E}{d P}=\frac{P}{E}\left(1+D x_{I}-F \frac{m_{I}^{2}}{E^{2}}\right),
$$

where

$$
D=4\left(\frac{\alpha_{S}}{\alpha_{I}}\right)^{-\kappa}+\frac{14 \kappa \alpha_{I}}{4 \pi}\left(\frac{\alpha_{S}}{\alpha_{I}}\right)^{1-\kappa}
$$

and

$$
F=\frac{7 \alpha_{I}}{4 \pi}\left(\frac{\alpha_{S}}{\alpha_{I}}\right)^{2 \tau+1}\left(\tau+7(\tau-\kappa) x_{I}\left(\frac{\alpha_{S}}{\alpha_{I}}\right)^{-\kappa}\right) .
$$

The point here is that the coefficients in the dispersion relation depend only on $\alpha_{S} / \alpha_{I}$ and therefore vary only logarithmically with the energy $E$. The outcome for the velocity of quark based particles is shown in Eq. (126). Omitting all terms $O\left(\alpha_{I}\right)$ that decrease logarithmically, we are left with the simple result

$$
v \simeq \frac{P}{E}\left(1+4 x_{I}\left(\frac{\alpha_{S}}{\alpha_{I}}\right)^{-\kappa}\right) .
$$

This is qualitatively different from LSV originating in higher derivative contributions to the QCD Lagrangian $[32,33]$ or spacetime foam models [34,35]. These are parametrized by large mass scales and suggest power law increases in energy. In our case, Eq. (129) suggests a slow logarithmic increase that we might expect to be more difficult to detect. However, were LSV to have been detected, the suggested energy dependence would distinguish this QCD model from such higher derivative models.

\section{CONCLUSIONS}

We have studied an $S U(N)$ QCD model with quarks in the fundamental representation and formulated the perturbation series to one loop with no restriction on the magnitude of the Lorentz symmetry breaking. In the particular case we studied, the LSV was due entirely to a mismatch between the light cones of the quarks and 
gluons. This is a consistent possibility if the light cones are generated by two metrics that are both invariant under the same subgroup of the Lorentz group that leaves a 4-vector, timelike in both metrics, invariant, a rotation group in fact. Similar results can be obtained with spacelike and lightlike vectors.

The renormalization group equation for the coupling constant $\alpha_{S}$ and the LSV parameter $b$ was obtained with the result, exhibited in Fig. 7, that initially $\alpha_{S}$ decreases with energy just as in the standard Lorentz symmetric case. However, $b$ departs from unity increasingly with energy, and this enhances the contribution of the quark vacuum polarization to the $\beta$-function for $\alpha_{S}$. The outcome is that at sufficiently high energy $\alpha_{S}$ ceases to decrease, reaches a minimum, and then increases again with energy. This constitutes the frustration of asymptotic freedom in QCD with LSV of the kind we have investigated. We suggest plausible values for the energy range $E>M_{I}$ we are investigating and the associated initial value $\alpha_{I}$ for the strong coupling. The outcome is that the frustration part of the RG trajectory for $\left(\alpha_{S}, b\right)$ is at energies many orders of magnitude greater than is accessible to accelerator experiments. It is well above the Planck mass. However, it is possible that part of the RG trajectory lying near the Lorentz symmetry line $b=1$ might be attainable in accelerator or cosmic ray observations. The effect on the dispersion relation of particles is through powers of $\alpha_{S}$ and hence is logarithmic in character and represents a kind of intrinsic LSV rather than one parametrized by higher derivative contributions to the Lagrangian.

There are many variations of the model that might be investigated such as increasing the number of quarks, varying the quark metrics in ways that induce more complex LSV associated with higher Petrov classes. Of course, one should also consider how these results relate to the full structure of the Standard Model and its extensions. Finally, it is worth noting that in the context of relatively weak LSV it may be possible to pursue a nonperturbative investigation of our model using the techniques of lattice QCD.

\section{ACKNOWLEDGMENTS}

This work has been partially supported by UK Science and Technologies Facilities Council consolidated Grant No. ST/P000681/1. I am grateful to R. R. Horgan for discussions concerning the potential relevance of lattice field theory calculations to evaluating LSV in gauge theories.
[1] M Schreck, Quantum field theory based on birefringent modified Maxwell theory, Phys. Rev. D 89, 085013 (2014).

[2] F. R. Klinkhammer and M. Schreck, Consistency of isotropic modified Maxwell theory: Microcausality and unitarity, Nucl. Phys. B848, 90 (2011).

[3] F. R. Klinkhammer and M. Schreck, Models for low energy Lorentz violation in the photon sector, Nucl. Phys. B856, 666 (2012).

[4] D. Collady and V. A. Kostelecký, Lorentz-violating extension of the standard model, Phys. Rev. D 58, 116002 (1998).

[5] V. A. Kostelecký, C. D. Lane, and A. G. M. Pickering, Oneloop renormalization of Lorentz-violating electrodynamics, Phys. Rev. D 65, 056006 (2002).

[6] R. Potting, M. Cambiaso, and R. Lehnert, Asymptotic states and renormalisation in Lorentz-violating quantum field theory, Phys. Rev. D 90, 065003 (2014).

[7] I. T. Drummond, Bimetric QED, Phys. Rev. D 95, 025006 (2017).

[8] I. T. Drummond, Lorentz symmetry breaking and causality, J. Phys. Conf. Ser. 952, 012001 (2018).

[9] Y. Itin, On light propagation in premetric electrodynamics, J. Phys. A 42, 475402 (2009).

[10] F. A. Asenjo, C. Erices, A. Gomberoff, S. A. Hojman, and A. Montecinos, Differential geometry approach to asymmetric transmission of light, Opt. Express 25, 26405 (2017).
[11] H. B. Nielsen and M. Ninomiya, $\beta$-function in a noncovariant Yang-Mills theory, Nucl. Phys. B141, 153 (1978).

[12] H. B. Nielsen and I. Picek, Lorentz non-invariance, Nucl. Phys. B211, 269 (1983).

[13] S. Chada and H. B. Nielsen, Lorentz invariance as a low energy phenomenon, Nucl. Phys. B217, 125 (1983).

[14] A. J. G. Carvalho, D. R. Granado, J. R. Nascimento, and A. Y. Petrov, Non-Abelian aether-like term in four dimensions, Eur. Phys. J. C 79, 817 (2019).

[15] D. J. Gross and F. Wilcek, Ultraviolet Behavior of NonAbelian Gauge Theories, Phys. Rev. Lett. 30, 1343 (1973).

[16] H. D. Politzer, Reliable Perturbative Results for Strong Interactions, Phys. Rev. Lett. 30, 1346 (1973).

[17] A. R. Vieira and N. Sherrill, Lorentz-violating running of coupling constants, arXiv:1911.07780.

[18] A. Z. Petrov, Classification of spaces defined by gravitational fields, Uch. Zapiski Kazan Gos. Univ. 114, 55 (1954).

[19] J. Stewart, Advanced General Relativity, Cambridge Monographs on Mathematical Physics (Cambridge University Press, Cambridge, England, 1991).

[20] P. O'Donnell, Introduction to 2-Spinors in General Relativity (World Scientific, Singapore, 2003).

[21] R. Penrose and W. Rindler, Spinors and Spacetime 2: Spinor and Twistor Methods in Spacetime Geometry (Cambrige University Press, Cambridge, England, 1986). 
[22] S. Coleman and S. Glashow, High-energy tests of Lorentz invariance, Phys. Rev. D 59, 116008 (1999).

[23] M. E. Peskin and D. V. Schroeder, An introduction to Quantum Field Theory (CRC Press, Boca Raton, Florida, 1995).

[24] G. 't Hooft and M. Veltman, Regularization and renormalization of gauge fields, Nucl. Phys. B44, 189 (1972).

[25] I. T. Drummond, Quantum field theory in a multimetric background, Phys. Rev. D 88, 025009 (2013).

[26] S. J. Brodsky A. Duer and G.F. Téramond, The QCD running coupling, Prog. Part. Nucl. Phys. 90, 1 (2016).

[27] D. W. Duke, W. A. Bardeen, A. J. Buras, and T. Muta, Deep inelastic scattering beyond the leading order in asymptotically free gauge theories, Phys. Rev. D 18, 3998 (1978).

[28] S. de Alwis, A. Eichhorn, A. Held, J. M. Pawlowski, M. Schiffer, and F. Versteegen, Asymptotic safety, string theory and weak gravity conjecture, Phys. Lett. B 798, 134991 (2019).
[29] A. Platania, A. Eichhorn, and M. Schiffer, Lorentz invariance violations in the interplay of quantum gravity with matter, arXiv:1911.10066.

[30] D. Collady and V. A. Kostelecký, Cross sections and Lorentz violation, Phys. Lett. B 511, 209 (2001).

[31] H. Fu and R. Lehnert, Moller scattering and Lorentzviolating Z bosons, Phys. Lett. B 762, 33 (2016).

[32] V. A. Kostelecký and M. Mewes, Electrodynamics with Lorentz-violating operators of arbitrary dimension, Phys. Rev. D 80, 015020 (2009).

[33] V. A. Kostelecký and Z. Li, Gauge field theories with Lorentz-violating operators of arbitrary dimension, Phys. Rev. D 99, 056016 (2019).

[34] N. E. Mavromatos, J. Ellis, and D. V. Nanopoulos, Derivation of a refractive index in stringy space-time foam model, Phys. Lett. B 665, 412 (2008).

[35] A.S. Sakharov J. Ellis, N.E. Mavromatos, and E. K. Sarkisyan-Grinbaum, Limits on Lorentz violation from multimessenger observations of TXS $0506+056$, Astrophys. J. 535, 139 (2000). 